Conference Report

\section{The 2016 Olten Meeting at the Basel Life Science Week}

\author{
Elsbeth Heinzelmann, \\ Science + Technology Journalist
}

Abstract: "This 'telephone' has too many shortcomings to be seriously considered as a means of communication. The device is inherently of no value to us." This was an internal memo written by Western Union in 1876. That's right. Without efficient knowledge sharing and technology transfer, even the best scientific development may prove to be a damp squib for a long time. The Basel Life Science Week was created in order to promote scientific and economic exchange and pave the way for innovative ideas. That's why NTN Swiss Biotech has moved its traditional 'Olten Meeting' to the Basel Life Science Week. It is the ideal setting for NTN Swiss Biotech and the School of Life Sciences FHNW to present innovative developments within its network of academic and industrial partners in the futureoriented disciplines of Molecular Diagnostics and Medicinal Chemistry. Short summaries of the key lectures are reported below.

Keywords: Cancer diagnostics - High-tech expression analysis . Infectious disease $\cdot$ Personalized medicine

\section{Part 1: Molecular Diagnostics \\ How To Detect Viral and Bacterial Infections and Genetic Disorders}

Molecular diagnostics is particularly on trend as this technology 'sniffs out' specific - potentially disease-related sequences in DNA or RNA. Clinical applications are numerous, ranging from infectious diseases and pharmacogenomics to oncology, genetic disease screening, human leukocytes, antigen typing and coagulation. During the Basel Life Science Week, from 19-23 September 2016, international speakers presented their topics under the motto 'Molecular Diagnostics brought by NTN Swiss Biotech'. The forum was chaired by Eric Kuebler and Daniel Gygax from the School of Life Sciences FHNW.

\section{Pioneer Performance in Gene Expression Assays}

How can we monitor hundreds of thousands of genes at once in a high-throughput system? Harper VanSteenhouse, director of BioSpyder Technologies Inc., has developed a novel, highly multiplexed, targeted RNA expression assay called TempO$\mathrm{Seq}^{\mathrm{TM}}$, which leverages the efficient use of existing NGS (nextgeneration sequencing) instruments for readout. Its unique attributes mean that it can be used in many high-throughput applications where RNA-Sequencing or other methods are too limited in performance or throughput or are cost-prohibitive. TempO-Seq ${ }^{\mathrm{TM}}$ achieves exceptionally high performance by utilizing annealing and ligation steps without the limitations of reverse transcription or preamplification. The assay generates a reduced-complexity barcoded and pooled NGS library, is robust to sequencing errors and minimizes necessary bioinformatics. It supports very high throughputs with $384+$ samples multiplexed per flowcell lane.

"The capture-free assay is simple to perform manually in a 96-well format in less than 8 hours using common laboratory equipment, including any PCR/qPCR instrument, and can be automated", explained VanSteenhouse. "As an example of TempO-Seq ${ }^{\mathrm{TM}}$ performance, we describe the highly sensitive and quantitative whole transcriptome and targeted surrogate panel gene expression profiling from $1 \mathrm{~mm}^{2}$ areas of $5 \mu \mathrm{m}$ formalin-fixed, paraffin-embedded (FFPE) sections of normal and cancerous tissue within the same prostate to identify disease biomarkers and mechanistic pathways. Both panels identified differentiating biomarkers and mechanistic pathways in prostate cancer."

With TempO-Seq ${ }^{\circledR}$, large cohorts of data take minutes to analyze, and pooling samples and multiplexing 1000s of genes makes sequencing extremely cost-effective, with up to 6,144 samples in one sequencing run. We gain deeper insights, as it is possible to analyze any number of targets while processing hundreds to millions of samples. The method is simple: there are no captures or bead-based cleanup steps, it is modular and scalable and it brings the power of targeted sequencing to highthroughput sample processing with the use of template-dependent oligo ligation.

http://biospyder.com

\section{The Fight against Drug-resistant Tuberculosis}

The WHO statistics are frightening: nearly half a million new cases of drug- resistant tuberculosis (DR-TB) emerge each year and, in certain countries, serious epidemics jeopardize progress. In 2014 alone, an estimated 190,000 people with DR-TB died. Dr. Christopher Gilpin, Senior Scientist, Global TB Programmes at WHO, explained the current situation and how his organization is fighting against this intractable disease with determination: the diagnosis and treatment of DR-TB are indeed a real challenge, but there are effective treatments available. "We call for early diagnosis and initiation of appropriate treatment of all persons of all ages with any form of drug-susceptible or drug-resistant TB", recalled Christopher Gilpin, who has over 20 years' experience as a microbiology scientist managing laboratories in Australia and the Middle East. This requires ensuring access to WHO-recommended rapid diagnostics that can detect TB early on in the disease process, along with universal access to drug susceptibility testing (DST) to detect drug-resistant forms. A number of laboratory tools are available to diagnose DRTB, including phenotypic culture-based DST and molecular methods. Molecular methods are a rapid alternative (results available in a single day) compared to culture-based DST, where results are often only available after several weeks. "Optimal and complementary use of the available diagnostic tools at the different levels of the tiered network of TB laboratories is critical. Accurate and timely diagnosis of DR-TB is essential to enable effective treatment and care of patients", explained Christopher Gilpin, who holds a doctorate in TB diagnostics and molecular epidemiology and a master of Public Health degree focused on tuberculosis and migration. Tools for sequencing and analyzing the genome for Mycobacterium tuberculosis in order to detect mutations associated with drug resistance have become widely 


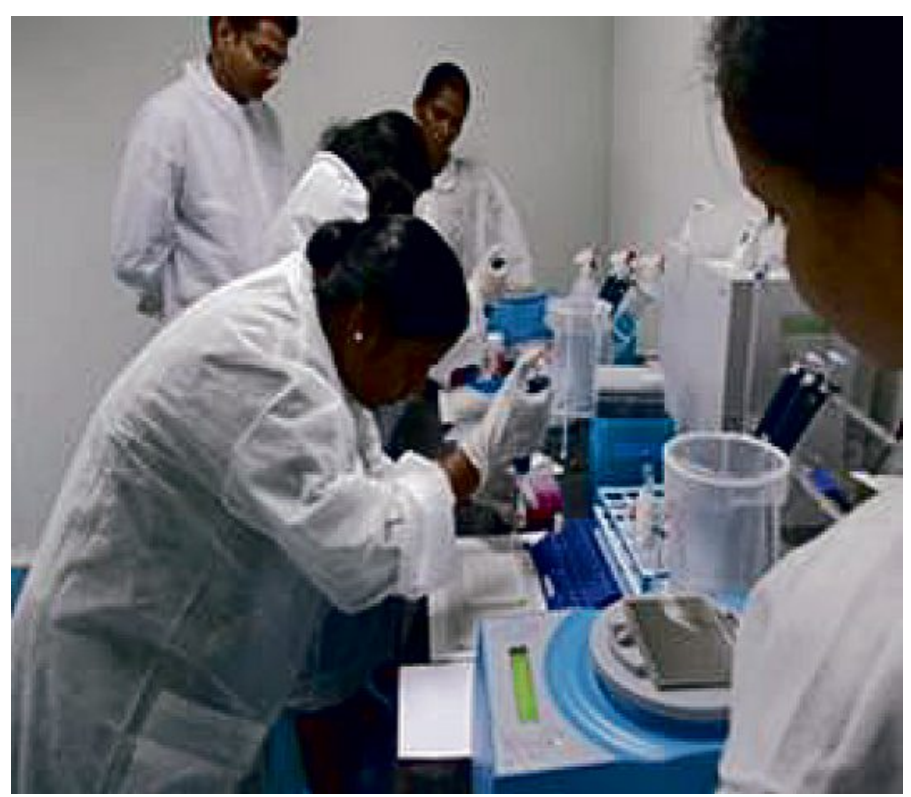

Diagnosis and treatment of drug-resistant tuberculosis are indeed a real challenge, but effective treatment is available. Source: WHO

accessible. "But greater understanding of the relationship between the mutations detected and patient outcomes is still needed for clinical decision making."

http://www.who.int/features/qa/79/en/

\section{Part 2: Medicinal Chemistry and Drug Discovery How To Create Pharmacologically Active and Safe Medicines}

Important topics in Medicinal Chemistry are the binding affinity as one of the major criteria in the prediction of potency for novel drug molecules. Our understanding of kinetic and thermodynamic parameters in drug-binding leads to advances in drug discovery. The forum provided an insight into new macrocyclic drug discoveries and therapeutic opportunities for peptides in oncology and diseases with unmet medical need. This forum was chaired by Gerhard Grundler and Daniel Gygax from the School of Life Sciences FHNW.

\section{The Discovery of LTX-315}

Bengt Erik Haug, Professor at the Department of Chemistry at the University of Bergen, is interested in the design and synthesis of biologically active peptides and peptidomimetics as well as the synthesis of marine natural products and analogues. Cationic antimicrobial peptides derived from host defence peptides (HDPs), which play an important role in the first line defence against pathogens for most species, have been targeted as novel antimicrobials and anticancer agents. "In many cases, the antimicrobial and cytotoxic activities come from peptidemembrane interactions implicating anionic constituents on bacterial cell surfaces and membranes in cancer cells", explained Haug. The membranolytic mode of action of cationic anticancer peptides includes the release of danger-associated molecular pattern molecules (DAMPs) and tumour antigens from cancer cells, resulting in regression of solid tumours and systemic tumour specific immune response (Ref).

"Thus, the concept of oncolytic peptides as a novel therapeutic modality has emerged as a promising immunotherapeutic strategy for cancer", said Haug, who worked at Lytix Biopharma for four years. "In our efforts to develop oncolytic peptides, initially through modification of the HDP bovine lactoferricin,

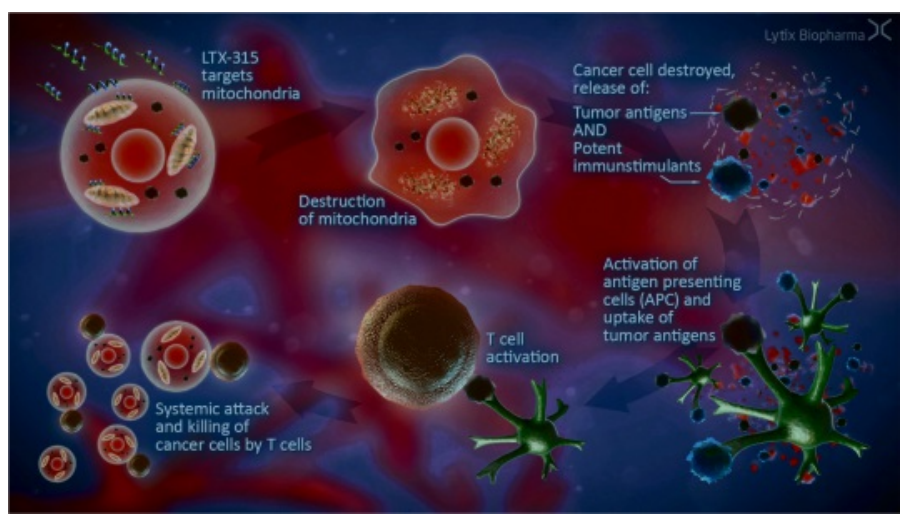

Preclinical studies show that LTX-315 has relatively high plasma protein binding abilities and a human plasma half-life in vitro of $160 \mathrm{~min}$. Lytix Biopharma AS selected LT-315 as a lead candidate as it displays superior anti-cancer activity. Source: Lytix Biopharma AS

we identified a series of chemically modified 9-mer cationic peptides with high cytotoxic activity against both drug-resistant and drug-sensitive cancer cells and lower toxicity towards normal cells."

Among these peptides, LTX-315 displayed superior anticancer activity and was selected as a lead candidate by Lytix Biopharma AS. Preclinical studies showed that LTX-315 has relatively high plasma protein binding abilities and a human plasma half-life in vitro of $160 \mathrm{~min}$. In addition, LTX-315 demonstrated relatively low ability to inhibit CYP450 enzymes and was metabolized into non-toxic metabolites. And Haug concluded: "LTX-315 is now in clinical development as a new anticancer agent for intra-tumoral administration by Lytix Biopharma, and is currently being evaluated in a phase I study."

http://www.uib.no / Bengt-Erik.Haug@uib.no

\section{Macrocycles - Panacea for unmet Medical Needs?}

The Swiss company Polyphor Ltd. is focusing on the discovery and development of macrocycle drugs for highly unmet medical needs. These macrocycles represent a new drug class that complements the classical small molecules and large

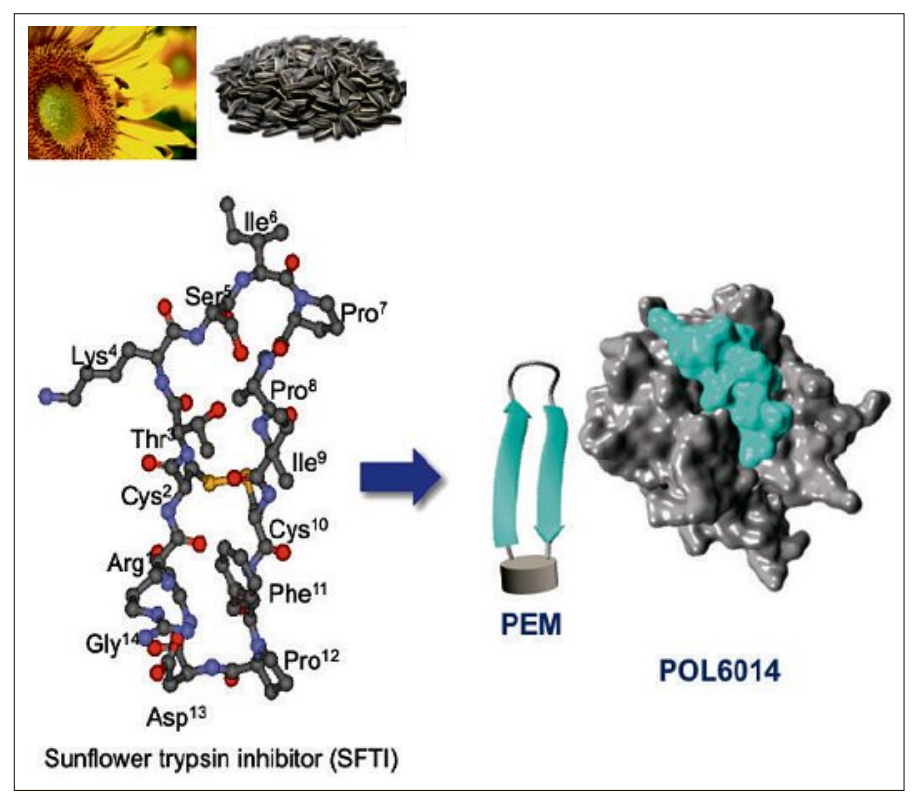

POL6014 is a novel, highly potent, selective and reversible inhibitor of human neutrophil elastase ( $\mathrm{hNE}$ ), a key target for the treatment of respiratory diseases like chronic obstructive pulmonary disease (COPD), cystic fibrosis (CF) and alpha-1-antitrypsin deficiency (AATD). Source: Polyphor Ltd. 
biopharmaceuticals. Within this strategy, Polyphor has established two complementary, fully proprietary macrocycle technologies, PEMfinder $^{\circledR}$ (macrocyclic peptides) and MacroFinder ${ }^{\circledR}$ (nonpeptidic macrocycles). As Daniel Obrecht, co-founder and Chief Scientific Officer explained, both technologies yield compounds that are synthetically accessible, amenable to a rapid and efficient optimization process. They have a proven potential to provide innovative drug candidates for complex target classes, including large surface protein-protein interactions.

The investigations carried out show innovative new findings: POL6014 is a highly potent and selective macrocyclic inhibitor of human neutrophil elastase (HNE) and is administered by aerosol inhalation. "POL6014 is currently in Phase I clinical development with the focus on cystic fibrosis (CF), non-cystic fibrosis bronchiectasis (NCFB) and other rare lung diseases", explained Daniel Obrecht, who spent many years at the Central Research Laboratories of Roche in Basel. "Our macrocycle pipeline also includes novel breakthrough antibiotics for the highlyselective treatment of serious infections caused by Pseudomonas aeruginosa, as well as a preclinical programme targeting multidrug-resistant Gram-negative ESKAPE pathogens."

The transition from bench to bedside is within reach: preclinical pharmacological studies reveal that POL6014 inhibits the aggressive, tissue-degrading enzyme neutrophil elastase in a highly potent, selective and reversible manner. After local application to the lungs by inhalation, POL6014 reaches high concentrations in the lung while the systemic exposure remains low, thus reducing the risk of side effects. Toxicology studies show that POL6014 is very well tolerated by aerosol administration. POL6014 is in Phase I clinical development.

www.polyphor.com / daniel.obrecht@polyphor.com

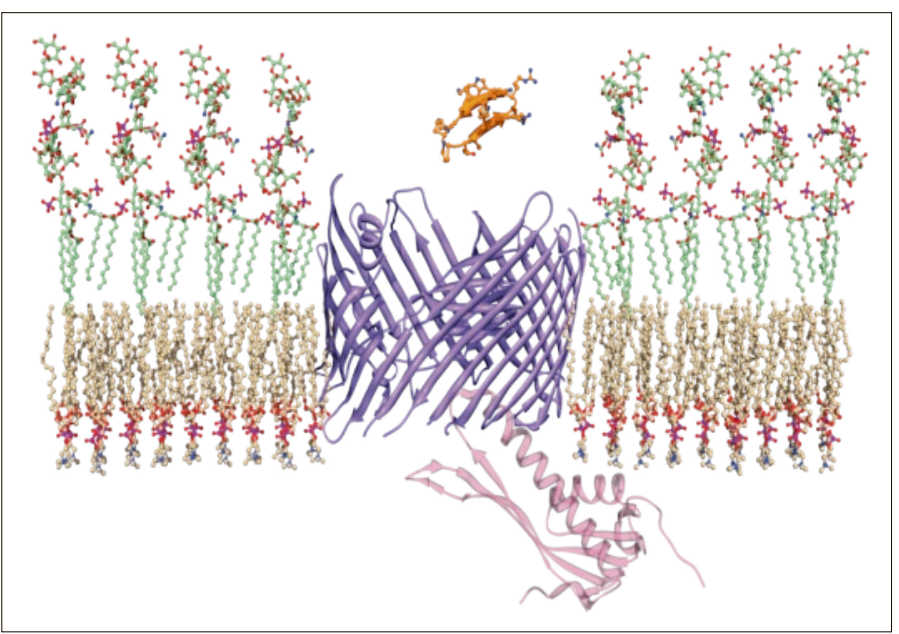

POL7080 is a Pseudomonas-specific antibiotic with a novel mode of action. Pseudomonas aeruginosa is a bacterium commonly found in the environment which can give rise to serious, life-threatening infections in humans and animals. Pseudomonas aeruginosa is regarded as an opportunistic pathogen, and serious infections in various types of tissue develop in patients with a compromised or reduced immune system, e.g. in case of AIDS or cancer treatment. Source: Polyphor Ltd.

The interest of the audience in both events at the Basel Life Science Week shows the need for closer links between academia and industry. Simply publishing research results in important journals provides no guarantee that the new findings will help us develop new procedures and products. First of all we have to develop new technologies. Researchers achieve this only through effective cooperation with innovative industrial partners. NTN Swiss Biotech paves the way and leads to success!

Your contact partner for inquiries:

Profesor Dr. Daniel Gygax

FHNW Muttenz

E-mail: daniel.gygax@fhnw.ch 